\title{
DIFERENCIAS EN LAS CALIFICACIONES DE ALUMNADO UNIVERSITARIO EN FUNCIÓN DE SU PARTICIPACIÓN EN UN PROGRAMA DE APRENDIZAJE-SERVICIO
}

Differences in university students' grades depending on their participation in a program of Service-Learning

Diferenças nas classificações de alunos universitários, em função da sua participação num programa de Aprendizagem-Serviço

Laura Cañadas (1)

$\mathrm{M}^{\mathrm{a}}$ Luisa Santos-Pastor (2)

Este trabajo se ha llevado a cabo dentro de los Proyectos de Innovación FPE_009.17_INN y FPE_013.18_IMP (Universidad Autónoma de Madrid).

(1) Departamento de Educación Física, Deporte y Motricidad Humana. Facultad de Formación de Profesorado y Educación. Universidad Autónoma de Madrid. Teléfono: +34 4975924. Correo electrónico: laura.cannadas@uam.es. ORCID: https://orcid.org/0000-0003-4179-9018

(2) Departamento de Educación Física, Deporte y Motricidad Humana. Facultad de Formación de Profesorado y Educación. Universidad Autónoma de Madrid. Teléfono: + 34 4973060. Correo electrónico: marisa.santos@uam.es

\section{Resumen}

El Aprendizaje-Servicio Universitario ha mostrado un impacto positivo en el aprendizaje del alumnado, sin embargo, la evidencia respecto a cómo afecta a las calificaciones de estos es escasa. Por tanto, en esta investigación se busca analizar las diferencias en las calificaciones de los estudiantes que participan en proyectos de aprendizaje-servicio respecto a los que eligen otros proyectos de aprendizaje. Participaron 194 alumnos del Grado en Ciencias de la Actividad Física y el Deporte, 54 de ellos participaban en proyectos de Aprendizaje-Servicio. Se recogieron las calificaciones finales en la asignatura, y las calificaciones en la tarea de AprendizajeServicio y en la tarea alternativa que el alumnado no participante realizaba. Los resultados muestran que aparecen diferencias significativas $(P<0.001)$ en las dos variables estudiadas, siendo el alumnado participante en Aprendizaje-Servicio el que mostró mejores resultados académicos. Las conclusiones indican que los estudiantes que mejores calificaciones obtienen son los que desarrollaron proyectos de AprendizajeServicio. 
Palabras clave: Aprendizaje-servicio; evaluación; calificación; rendimiento académico

\begin{abstract}
University Service-Learning has shown a positive impact in students' learning, but there is small evidence about how it affects students' grades. So, this research aims to analyze differences in the grades of students participating in service-learning projects compared to those who choose other learning projects. One hundred and ninety-four students from Physical Activity and Sport Sciences Degree participated, 54 of them participated in the Service-Learning Program. The final grades in the subject were collected, as well as the grades in the Learning-Service task and in the alternative task that non-participating students performed. Results show significant differences $(P<0.001)$ in both variables studied, being the students that participated in Service-Learning the one that show better academic results. Conclusions indicated that students with better grades are the ones that participated in Service-Learning Program.
\end{abstract}

Keywords: Service-learning; assessment; grading; academic achievement

\title{
Resumo
}

A Aprendizagem-Serviço na Universidade mostrou um impacto positivo na aprendizagem dos alunos, no entanto, as evidências sobre como isso afeta as classificações dos alunos são escassas. Neste sentido, esta investigação pretende analisar as diferenças nas notas entre os alunos que participam em projetos de aprendizagemserviço em comparação com aqueles que escolhem outros projetos de aprendizagem. Participaram 194 alunos participaram da Licenciatura em Ciências da Atividade Física e Desporto, sendo que 54 deles participaram em projetos de Aprendizagem-Serviço. Foram recolhidas as notas finais na disciplina, as notas na tarefa de AprendizagemServiço e na tarefa alternativa que os alunos não participantes fizeram. Os resultados mostram diferenças significativas aparecem $(P<0.001)$ nas duas variáveis estudadas, sendo os alunos participantes na Aprendizagem-Serviço, os que apresentaram melhores resultados académicos. As conclusões indicam que os alunos que obtêm as melhores notas são aqueles que desenvolvem projetos de Aprendizagem-Serviço.

Palavras-chave: Aprendizagem-serviço; avaliação; classificação; rendimento académico

Diferencias en las calificaciones de alumnado universitario en función de su participación en un programa de aprendizaje-servicio 


\section{Introducción}

El Aprendizaje-Servicio (ApS) como enfoque metodológico en Educación Superior contribuye a lograr una docencia e investigación de calidad. Diferentes estudios avalan su efectividad para el aprendizaje competencial del estudiante (Ruiz-Corbella \& GarcíaGutiérrez, 2018). El ApS necesita de un proceso sistematizado, bien articulado y coherentemente organizado (Uruñuela, 2018). La evaluación formativa en los proyectos de ApS es una herramienta para guiar y asesorar los procesos de enseñanza-aprendizaje de los estudiantes, favoreciendo el aprendizaje, mejorar la motivación y la reflexión del estudiante. Este tipo de evaluación exige un proceso de trabajo continuo del estudiante, y de supervisión constante del profesorado. Todo parece indicar que, los procesos de seguimiento de los proyectos de ApS, repercuten en el aprendizaje del estudiante, pudiendo mejorar sus resultados académicos respecto a otras formas de evaluación tradicionales. Sin embargo, aún no existen muchos estudios que avalen estas conclusiones (Deeley, 2016; Mella-Núñez, Santos-Rego \& Malheiro-Gutiérrez, 2015). Por tanto, parece necesario profundizar en esta temática.

Así, en esta investigación nuestro objetivo es valorar si existen diferencias en las calificaciones finales de materia del alumnado universitario en función de si participaron o no en un programa de $\mathrm{ApS}$; y valorar si existen diferencias en el porcentaje de calificación obtenido sobre la calificación asignada a la tarea ApS/tarea alternativa en función de su participación o no en el programa de ApS.

\section{Método}

En esta investigación se llevó a cabo un estudio transversal. La muestra se seleccionó por facilidad de acceso a la misma, siendo alumnos y alumnas que cursaban materias donde se llevaban a cabo procesos de ApS. Participaron 194 estudiantes que se encontraban matriculados en algunas de estas materias del Grado en Ciencias de la Actividad Física y el Deporte en la Universidad Autónoma de Madrid: Expresión Corporal y Deportes Colectivos y su Didáctica I (Hockey) de $1^{\circ}$ de Grado; Actividades Físico-Deportivas en el Medio Natural y Deportes Individuales y su Didáctica I (Atletismo) de $2^{\circ}$ de Grado. Se recogieron las calificaciones finales en la asignatura, y las calificaciones en la tarea de ApS y en la tarea alternativa que el alumnado que no participaba debía realizar. Como el porcentaje de la nota final asignado a esta tarea era diferente en función de la materia, se calculó el porcentaje que suponía la nota sobre la 
puntuación asignada a esa tarea (calificación obtenida en la tarea*100)/puntuación asignada a la tarea en cada materia). Los datos se recogieron en el curso 2017/2018. Los análisis estadísticos se realizaron con el SPSS v.21. Tras comprobar que los datos no presentaban una distribución normal, se utilizó la $U$ de Mann-Whitney para valorar las diferencias en las calificaciones entre el alumnado participante y el no participante en ApS. El nivel de significación se estableció en $P<0.05$.

\section{Resultados y discusión}

En la Tabla 1 se presentan los resultados relativos a las diferencias entre el alumnado participante y no participante en el proyecto de ApS en las calificaciones finales de la materia y en el valor medio del porcentaje obtenido sobre la calificación asignada a la tarea ApS/tarea alternativa.

Aparecen diferencias significativas $(p<0.001)$ entre los grupos tanto en el porcentaje sobre la calificación asignada a la tarea como sobre las calificaciones finales, siendo el grupo de estudiantes que participaron en ApS los que obtienen valores más altos en ambas variables. En el caso del porcentaje sobre la calificación asignada a la tarea, el alumnado participante en ApS tiene más de un 95\% de acierto de media, mientras que los no participantes en ApS no llegan al 70\%. En la calificación final de la materia el alumnado participante en ApS obtiene de media un notable $(\mathrm{M}=8.08)$ mientras que los no participantes se quedan en un bien alto $(M=6.75)$.

Tabla 1.

Diferencias en las variables estudiadas entre los participantes y los no participantes en propuestas de ApS

\begin{tabular}{|c|c|c|c|}
\hline & Participantes ApS & No participantes ApS & $P$ \\
\hline$n$, & 54 & 133 & \\
\hline $\begin{array}{l}\text { Porcentaje sobre la calificación } \\
\text { asignada a la tarea }\end{array}$ & $95.5(9.10)$ & $69.58(19.40)$ & $<0.001$ \\
\hline Calificación final de la materia & $8.08(1.08)$ & $6.75(1.86)$ & $<0.001$ \\
\hline
\end{tabular}

Nota: En negrita diferencias significativas

Por tanto, esta investigación muestra que el alumnado participante en ApS obtiene mejores resultados académicos que aquellos que no participaron, como también han constatado investigaciones previas (Mella-Núñez et al., 2015; Strage, 2000). Algunas investigaciones, como la de Astin, Vogelgesang, Ikeda, y Yee (2000) proponen que estas diferencias pueden ser debidas a que este tipo de actividades requiera menos 
esfuerzo por parte del alumnado, resultándoles más fácil obtener una alta calificación. Sin embargo, otros estudios, como el de Mella-Nuñez et al. (2015) abogan porque estas diferencias pueden ser producidas porque el mayor compromiso académico que requieren estas actividades pueda desembocar en un mayor rendimiento académico.

Por otra parte, también cabe destacar que menos de la mitad del alumnado elige la opción del ApS, pudiendo deberse esto al desconocimiento de lo que es esta metodología por parte del alumnado universitario y el miedo a que incorporarse al mismo suponga más trabajo para ellos.

\section{Conclusiones}

Esta investigación muestra que el alumnado participante en las propuestas de ApS obtiene mejores resultados académicos tanto en la tarea concreta con la que se evalúan las competencias puestas en el ApS como en la tarea alternativa que debe evaluar las mismas competencias a través de una propuesta diferente, como en la calificación final de las materias. Sin embargo, la investigación sobre esta temática aún es escasa, y hacen falta más estudios que profundicen en el rendimiento académico del alumnado participante en ApS.

\section{Referencias}

Astin, A. W., Vogelgesang, L. J., Ikeda, E. K., \& Yee, J. A. (2000). How ServiceLearning affects students. Los Angeles: Higher Education Research Institute. Recuperado de http://heri.ucla.edu/pdfs/hslas/hslas.pdf

Deeley, S. (2016). El aprendizaje-Servicio en educación superior. Teoría, práctica y perspectiva crítica. Madrid: Narcea.

Mella-Núñez, I., Santos-Rego, M.A., \& Malheiro-Gutiérrez, X.M. (2015). AprendizajeServicio y rendimiento académico del alumnado universitario. Revista de Estudios e Investigación en Psicología y Educación, Extra (12), 35-39. DOI: 10.17979/reipe.2015.0.12.569

Ruiz-Corbella, M., \& García-Gutiérrez, J. (2019). Aprendizaje-Servicio. Los retos de la evaluación. Madrid. Narcea.

Strage, A. A. (2000). Service-Learning: Enhancing student learning outcomes in a college-level lecture course. Michigan Journal of Community Service Learning, $7,5-13$. 
Uruñuela, P.M. (2018). Metodología del Aprendizaje-Servicio. Aprender mejorando el mundo. Madrid. Narcea. 